\title{
A Note on the Boundedness and the Stability of Solutions of Certain Third-Order Differential Equations. $(*)$
}

\author{
H. O. TeJumola (a Ibadan, Nigeria)
}

Summary. - This paper establishes conditions under which solutions of equations of the form (1.1) are bounded or tend to zero as $t \rightarrow \infty$.

1. - This note is concerned with real third-order differential equations of the form

$$
\dddot{x}+f(x, \dot{x}, \ddot{x}) \ddot{x}+g(x, \dot{x})+h(x)=p(t, x, \dot{x}, \ddot{x})
$$

in which $f, g, h$ and $p$ depend only on the arguments explicitly displayed, and the dots denote differentiation with respect to $t$. It will be assumed throughout what follows that $h^{\prime}(x), g(x, y)$ and $p(t, x, y, z)$ are continuous in their respective arguments and that $\partial g / \partial x(x, y), \partial f / \partial x(x, y, z)$ and $\partial f / \partial z(x, y, z)$ exist and are continuous for all $x, y$ and $z$.

The problem of interest here is to investigate conditions under which all solutions of (1.1) are bounded or tend to zero as $t \rightarrow \infty$. This problem has received considerable attention from a number of authors (EzeILo [1], HaRrow [3], REIssing [4], TejumLoA [5]) for various special cases of (1.1). The object of the present note is to extend these earlier results to equations of the form (1.1).

Our stability result which is motivated by EzeILo's result [1; Theorem 1] is as follows.

TheOREM 1. - Suppose that $g(x, 0)=h(0)=0$ and that

(i) there are constants $a, a^{\prime}, b, b^{\prime}$ such that $a^{\prime} \geqslant f(x, y, z)>a>0$ for all $x, y, z$ and, $b^{\prime} \geqslant g(x, y) \mid y \geqslant b>0$ for $y \neq 0$ and all $x$; constants;

(ii) $h(x) / x \geqslant \delta(x \neq 0)$ and $h^{\prime}(x) \leqslant c$ for all $x$, where $\delta>0, c, 0<c<a b$, are

(iii) $y(\partial f / \partial x)(x, y, 0) \leqslant 0, y(\partial f / \partial z)(x, y, z) \geqslant 0,(\partial g / \partial x)(x, y) \leqslant 0$ for all $x, y$ and $z$;

(iv) the function $p$ satisfies

$$
|p(t, x, y, z)| \leqslant \varphi_{1}(t)+\varphi_{2}(t)\left(x^{2}+y^{2}+z^{2}\right)^{\frac{1}{2} x}+\Delta\left(x^{2}+y^{2}+z^{2}\right)^{\frac{1}{2}}
$$

(*) Entrata in Redazione il 14 aprile 1971.

5 - Annali di Matematica 
where $\alpha$ and $\Delta$ are constants such that $0 \leqslant a<1, A \geqslant 0$, and the functions $\varphi_{1}(t) \geqslant 0$, $\varphi_{2} \geqslant 0$ satisfy

$$
\int_{t}^{t+e} \varphi_{1}^{r}(\tau) d \tau \rightarrow 0 \text { and } \int_{t}^{t+e}\left\{\varphi_{2}(\tau)\right\}^{r(1-\alpha)^{-1}} d \tau \rightarrow 0
$$

as $t \rightarrow \infty$, for some fixed constants $r(1 \leqslant r \leqslant 2)$ and $\varrho>0$. Then there exists a finite constant $\mu=\mu\left(a, a^{\prime}, b, b^{\prime}, c, \delta\right)>0$ such that if $A \leqslant \mu$ then every solution $x(t)$ of (1.1) satisfies

$$
x^{2}(t)+\dot{x}^{2}(t)+\ddot{x}^{2}(t) \rightarrow 0 \quad \text { as } t \rightarrow 0
$$

In view of Ezeilo's result [1; Theorem 1] for the special case in which $f$ depends only on $x$ and $\dot{x}$ and $g$ only on $\dot{x}$ our present conditions of $f$ and $g$ may seem rather restrictive. Here, however, the right hand side of (1.2) involves terms in $x$ explicity, a situation which could not be attained in [1] (see particularly the remark concerning $|p(t, x, y, z)|$ on page 234 of [1]). Besides, our condition (1.3) is much weaker than the corresponding one 1.2(1) in [1].

Also in the special case when $p \equiv p(t)$ satisfies

$$
\max |p(t)|<\infty \text { for all } t \leqslant t_{0} \text { and } \int_{t_{\mathrm{e}}}^{\infty}|p(t)|^{k} d t<\infty \quad(1 \leqslant k \leqslant 2),
$$

Harrow's result [3; Theorem 1] gives that every solution $x(t)$ of (1.1) satisfies (1.4). Theorem 1 includes and generalizes this stability result of Harrow.

THEOREM 2. - Suppose that

(i) there are constants, $a, a^{\prime}$ such that $0<a \leqslant f(x, y, z) \leqslant a^{\prime}$ for all $x, y$ and $z$;

(ii) there are positive constants $b, b^{\prime}, y_{0}, A$ such that

$$
b \leqslant g(x, y) / y \leqslant b^{\prime}\left(|y| \geqslant y_{0}\right) \quad \text { and } \quad \max _{|y| \leqslant y_{e}}|g(x, y)| \leqslant A \text { for all } x
$$

(iii) there are constants $x_{0}>0, \delta>0$ and 0 satisfiyng $0<0<a b$, such that

$$
h(x) / x \geqslant \delta \text { and } h^{\prime}(x) \leqslant 0 \text { for }|x| \geqslant x_{0}
$$

(iv) $f(x, y, z)$ and $g(x, y)$ satisfy hypothesis (iii) of Theorem 1 ;

(v) $p(t, x, y, z)$ satisfies

$$
|p(t, x, y, z)| \leqslant \Delta_{0}+\Delta_{1}\left(x^{2}+y^{2}+z^{2}\right)^{\frac{1}{2}}
$$


for all $t, x, y$ and $z$ where $\Delta_{0} \geqslant 0, \Delta_{1} \geqslant 0$ are constants. Then there exists a finite constant $\varepsilon=\varepsilon\left(a, a^{\prime}, b, b^{\prime}, c, \delta, x_{0}, y_{0}, A\right)>0$ such that if $\Delta_{1} \leqslant \varepsilon$ then every solution $x(t)$ of (1.1) ultimately satisfies

$$
x^{2}(t)+\dot{x}^{2}(t)+\ddot{x}^{2}(t) \leqslant D
$$

where $D$ is a finite constant whose magnitude depends only on $a, a^{\prime}, b, b^{\prime}, c, \delta, x_{0}, \Delta_{0}$, $\Delta_{1}, A$ and $h$.

The main feature of this result is the condition (1.6) which admits of forcing functions $p=p(t, x, y, z)$ which may be unbounded for arbitrarily large $x^{2}+y^{2}+z^{2}$. The theorem extends the boundedness results of ReIssing [4] and TeJunnon [5]. Theorem 2 also includes and generalizes the boundedness result obtained when [3; Theorem 1] is specialized to the case $|p(t)|$ bounded (see, in particular, remark on page 588 of $[3])$.

\section{2. - Some preliminaries.}

We shall use $D$ 's for positive constants whose magnitudes depend only on the constants appearing in the statement of the theorems as well as on the function $h$. The unnumbered $D$ 's are not necessarily the same in each place of occurrence, but the Ds: $D_{1}, D_{2}, D_{3}, \ldots$ with suffixes attached preserve their identities throughout this paper.

When it comes to the actual proof of Theorems 1 and 2 considerable use will be made of the function $W=W(x, y, z)$ defined (as in [3]) by

$$
W=W_{1}+W_{2}+W_{3},
$$

where

$$
\begin{aligned}
& 2 W_{1}=2 \int_{0}^{x} h(\xi) d \xi+2 \int_{0}^{y} \eta f(x, \eta, 0) d \eta+2 \delta_{1} \int_{0}^{y} g(x, \eta) d \eta+\delta_{1} z^{2}+2 y z+2 \delta_{1} y h(x), \\
& 2 W_{2}=\delta_{2} b x^{2}+2 a \int_{0}^{x} h(\xi) d \xi+\left(a^{2}-\delta_{2}\right) y^{2}+2 \int_{0}^{y} g(x, \eta) d \eta+z^{2}+2 a \delta_{2} x y+ \\
& +2 \delta_{2} x z+2 a y z+2 y h(x),
\end{aligned}
$$

$$
2 W_{3}=2 a \int_{0}^{y} \eta f(x, \eta, 0) d \eta-a^{2} y^{2}
$$

and $\delta_{1}>0, \delta_{2}>0$ are two constants chosen (as is possible since $a b>c>0$ ) such 
that

$$
a^{-1}<\delta_{1}<e^{-1} b, \quad a b-e>a \delta_{2}>0 \quad \text { and } \quad \delta>\left\{\left(b^{\prime}-b\right)+\left(a^{\prime}-a\right)\right\} \frac{\delta_{2}}{4} .
$$

The following two properties of $W$ will be useful.

Leinma 1. - Subject to the conditions of Theorem 1 there are constants $D_{1}, D_{2}$ sueh that

$$
D_{1}\left(x^{2}+y^{2}+z^{2}\right) \leqslant W(x, y, z) \leqslant D_{2}\left(x^{2}+y^{2}+z^{2}\right)
$$

for all $x, y$ and $z$.

Lemua 2. - Subject to the conditions of Theorem $2, W=W(x, y, z)$ satisfies, for all $x, y$ and $z$,

$$
W>D_{1}\left(x^{2}+y^{2}+z^{2}\right)-D
$$

for some constant $D$.

Proof of Lemara 1. - The right-hand side inequality in (2.6) follows from (2.1)-(2.4) and the fact that hypotheses (i) and (ii) of Theorem 1 imply that

$$
\begin{gathered}
\delta|x| \leqslant|h(x)| \leqslant c|x|, 0 \leqslant \int_{0}^{x} h(\xi) d \xi \leqslant \frac{1}{2} c x^{2} \\
a y^{2} \leqslant 2 \int_{0}^{x} \eta f(x, \eta, 0) d \eta \leqslant a^{\prime} y^{2} \text { and } b y^{2} \leqslant 2 \int_{0}^{y} g(x, \eta) d \eta \leqslant b^{\prime} y^{2} .
\end{gathered}
$$

To verify the left-hand inequality in (2.6), we use the fact that $2 \int_{0}^{y} \eta f(x, \eta, 0) \geqslant a y^{2}$ for all $x, y$ and $z$ to obtain

$$
\begin{gathered}
2 W \geqslant \delta_{2}\left(b-\delta_{2}\right) x^{2}+\left\{\left(a-\delta_{1}^{-1}\right)+\left(b-\delta_{2}-a^{-1} c\right)\right\} y^{2}+\delta_{1}\left(z+\delta_{1}^{-1} y\right)^{2}+ \\
+\left(z+a y+\delta_{2} x\right)^{2}+b \delta_{1}\left\{y+b^{-1} h(x)\right\}^{2}+a^{-1} c\left\{y+c^{-1} a h(x)\right\}^{2}+ \\
+\Phi_{1}(x)+a \Phi_{2}(x)+2\left(\delta_{1}+1\right) \Phi_{3}(y),
\end{gathered}
$$

where

$$
\Phi_{1}(x)=2 \int_{0}^{x} h(\xi) d \xi-b^{-1} \delta_{1} h^{2}(x), \Phi_{2}(x)=2 \int_{0}^{x} h(\xi) d \xi-e^{-1} h^{2}(x)
$$

and

$$
\Phi_{s}(y)=\int_{0}^{y}\{g(x, \eta)-b \eta\} d \eta
$$


Since $\Phi_{1}(x)$ can be rewritten

$$
\Phi_{1}(x)=2 \int_{0}^{x}\left\{1-b^{-1} \delta_{1} h^{\prime}(\xi)\right\} h(\xi) d \xi-\delta_{1} b^{-1}\{h(0)\}^{2}
$$

and $h(0)=0$, it is clear from hypotheses (ii) of Theorem 1 and $(2.5)$ that $\Phi_{1}(x) \geqslant 0$. Similarly $\Phi_{2}(x) \geqslant 0$ and, $\Phi_{3}(y) \geqslant 0$ by hypothesis (i). Thus, by $(2.8)$,

$$
\begin{array}{r}
2 W>\delta_{2}\left(b-\delta_{2}\right) x^{2}+\left\{\left(a-\delta_{1}^{-1}\right)+\left(b-\delta_{2}-a_{1}^{-1} c\right)\right\} y^{2}+\delta_{1}\left(z+\delta_{1}^{-1} y\right)^{2}+ \\
+\left(z+a y+\delta_{2} x\right)^{2}
\end{array}
$$

Since $\delta_{2}\left(b-\delta_{2}\right), a-\delta_{1}^{-1}, b-\delta_{2}-a^{-1} c$ and $\delta_{1}$ are all positive, by (2.5), the left-hand side inequality in (2.6) follows provided $D_{1}$ is small enough.

Proof of LEMMA 2. - Note from $(1.6),(2.5),(2.9)$ and the fact that $h$ is continuous that

$$
\Phi_{1}(x) \geqslant-D \quad \text { for all } x
$$

Similarly, $\Phi_{2}(x) \geqslant-D$ for all $x$. Also, since $g(x, y) \mid y \geqslant b\left(|y| \geqslant y_{0}\right)$ and $\max _{|y| \leqslant y_{0}}|g(x, y)| \leqslant A$, it is evident that $\Phi_{3}(y) \geqslant-D$ for all $y$. Lemma 2 therefore follows from $(2.8)$ and $(2.10)$.

\section{3. - Proof of Theorem 1 .}

Consider, instead of (1.1), the equivalent system

$$
\dot{x}=y, \dot{y}=z, \dot{z}=-f(x, y, z) z-g(x, y)-h(x)+p(t, x, y, z)
$$

obtained from (1.1) on setting $\dot{x}=y, \dot{y}=z$. The result (1.4) will follow once it can be shown that every solution $(x(t), y(t), z(t))$ of $(3.1)$ satisfies

$$
x^{2}(t)+y^{2}(t)+z^{2}(t) \rightarrow 0 \quad \text { as } t \rightarrow \infty
$$

Let $(x, y, z)$ denote an arbitrary solution $(x(t), y(t), z(t))$ of $(3.1)$ and set $\dot{W}=$ $=(d / d t) W(x(t), y(t), z(t))$ where $W$ is the function (2.1). Then by proceeding as in [3; Lemma 2] it can be shown that there are constants $D_{\mathrm{a}}, D_{4}$ such that

$$
\dot{W} \leqslant-2 D_{4}\left(x^{2}+y^{2}+z^{2}\right)+D_{3}\left(x^{2}+y^{2}+z^{2}\right)^{\frac{1}{2}}|p(t, x, y, z)|
$$


To simplify notations set $u^{2}=x^{2}+y^{2}+z^{2}$. Then, by (1.2),

$$
\begin{aligned}
\dot{W} & \leqslant-2 D_{4} u^{2}+D_{3}\left\{\varphi_{1} u+\varphi_{2} u^{\alpha+1}+\Delta u^{2}\right\} \\
& =-\left(D_{4}-\Delta D_{3}\right) u^{2}+\left(D_{3} \varphi_{1} u-\frac{1}{2} D_{4} u^{2}\right)+\left(D_{3} \varphi_{2} u^{\alpha+1}-\frac{1}{2} D_{4} u^{2}\right) \\
& \equiv-\left(D_{4}-\Delta D_{3}\right) u^{2}+\theta_{1}+\theta_{2} .
\end{aligned}
$$

Let $r$ be any constant such that $1 \leqslant r \leqslant 2$ and set $\lambda=2-r$ so that $0 \leqslant \lambda \leqslant 1$. We show that

$$
\theta_{2} \leqslant D_{5} u^{\lambda}\left\{p_{2}\right\}^{(2-\lambda)(1-\alpha)^{-2}} \quad \text { for all } t
$$

To this end rewrite $\theta_{2}$ (as possible) in the form

$$
\theta_{2}=u^{\lambda} \theta_{*},
$$

where $\theta_{*}=u^{(\alpha+1-\lambda)}\left(D_{3} \varphi_{2}-\frac{1}{2} D_{4} u^{(1-\alpha)}\right)$. If $D_{3} \varphi_{2} \leqslant \frac{1}{2} D_{4} u^{(1-\alpha)}$ for all $t$, then (3.4) holds since $\theta_{*} \leqslant 0$ in this case. On the other hand, if $D_{3} \varphi_{2}>\frac{1}{2} D_{4} u^{(1-\alpha)}$ for all $t$, then $u<\left(2 D_{3} D_{4}^{-1} \varphi_{2}\right)^{(1-\alpha)^{-1}}$ and so

$$
\begin{aligned}
\theta_{*} & \leqslant D_{3} \varphi_{2} u^{(\alpha+1-\lambda)} \\
& \leqslant D_{3} \varphi_{2}\left(2 D_{3} D_{4}^{-1} \varphi_{2}\right)^{(\alpha+1-\lambda)(\alpha-\alpha)^{-1}} \\
& =D_{5} \varphi_{2}^{(2-\lambda)(1-\alpha)^{-1}}
\end{aligned}
$$

which, in view of (3.5), verifies (3.4) with $D_{5}=D_{3}\left(2 D_{3} D_{4}^{-1}\right)^{(\alpha+1-\lambda)(1-\alpha)^{-1}}$. In a similar way, it can be shown that for some constant $D_{6}$,

$$
\theta_{1} \leqslant D_{8} u^{\lambda}\left\{\varphi_{1}\right\}^{(2-\lambda)} \text { for all } t \text {. }
$$

From (3.3) it therefore follows that

$$
\dot{W} \leqslant-\left(D_{4}-\Delta D_{3}\right) u^{2}+D_{7} u^{(2-r)}\left\{\varphi_{1}^{\tau}+\varphi_{2}^{r(1-\alpha)^{-1}}\right\}
$$

where $r=2-\lambda$ and $D_{7}=\max \left(D_{5}, D_{6}\right)$.

Now fix $\Delta$ so that

$$
\Delta \leqslant \mu, \mu \equiv \frac{1}{2} D_{4} D_{3}^{-1} .
$$

Then since $u^{2}=x^{2}+y^{2}+z^{2}$, we have, in view of $(2.6)$, that

$$
\dot{W} \leqslant-D_{8} W+D_{9} W^{\frac{1}{(2-n)}}\left\{\varphi_{1}^{r}+\varphi_{2}^{r(1-\alpha)^{-1}}\right\}
$$


where $D_{8}=\frac{1}{2} D_{4} D_{3}^{-1}$ and $D_{9}=D_{7} D_{1}^{t(2-r)}$. By setting $W=V^{\left(\frac{1}{3}\right)}$ and integrating the resulting inequality in $V$ between $t_{0} \geqslant 0$ and $t$ the rest of the proof which leads to the result

$$
W(x(t), y(t), z(t)) \rightarrow 0 \quad \text { as } t \rightarrow \infty
$$

can be carried out in exactly the same way as in $[1 ; \S 3.2]$. Thus $(3.3)$ follows, by (2.6), and Theorem 1 is therefore proved with $\mu$ given by (3.6).

\section{4. - Proof of Theorem 2.}

Consider the function $U=U(x, y, z)$ defined by

$$
U=W-B y \chi(x)
$$

where $W$ is the function (2.1),

$$
B=\frac{8 C}{\pi}\left(1+2^{-\frac{1}{2}} \delta_{1}\right), \quad C \equiv \max _{|x| \leqslant x_{0}}\left|h^{\prime}(x)\right|,
$$

and $\chi$ is a differentiable function of $x$ defined by

$$
\chi(x)=\left\{\begin{array}{ll}
\operatorname{sgn} x, & \text { if }|x|>2 x_{0} \\
\sin \left(\frac{\pi x}{4 x_{0}}\right), & \text { if }|x| \leqslant 2 x_{0}
\end{array}\right\}
$$

Since $|\chi(x)| \leqslant 1$, definition (4.1) and Lemma 2 yield

$$
U \geqslant D_{1}\left(x^{2}+y^{2}+z^{2}\right)-B|y|-D_{3}
$$

so that

$$
U(x, y, z) \rightarrow+\infty \quad \text { as } x^{2}+y^{2}+z^{2} \rightarrow \infty
$$

Since $U=U(x, y, z)$ is continuous, (4.4) implies the existence of a constant $D_{10}$ such that for all $x, y$ and $z$

$$
U(x, y, z) \geqslant-D_{10}
$$

Consider now the system (3.1) and let $(x, y, z)$ denote any solution $(x(t), y(t), z(t))$ of (3.1). As before set $\dot{U}=(d / d t) U(x(t), y(t), z(t))$. To conclude the proof of the theorem it would suffice now to verify the existence of a constant $D_{11}$ such that

$$
\dot{U} \leqslant-1 \text { provided } x^{2}+y^{2}+z^{2} \geqslant D_{\mathbf{1 1}} \text {. }
$$


For once (4.6) is established it can be shown as in $[2 ; \S 3]$ that the results $(4.4),(4.5)$ and (4.6) imply the existence of a constant $D_{12}$ such that

$$
x^{2}+y^{2}+z^{2} \leqslant D_{12}
$$

for all $t \geqslant t_{0}\left(0<t_{0}<\infty\right)$, and this verifies (1.8).

It is clear from (4.1), (4.2) and (3.1) that

$$
\dot{U}=\dot{W}-\frac{8 C}{\pi}\left(1+\delta_{1} / \sqrt{2}\right) y^{2} \chi^{\prime}(x)-B z \chi(x)
$$

The expression for $\dot{W}$ can be obtained from (2.1)-(2.4). Indeed, if as in [3] we use the fact arising from hypothesis (iv) of Theorem 2 that

$$
y \int_{0}^{y} \eta \frac{\partial f}{\partial x}(x, \eta, 0) d \eta \leqslant 0, \quad y \int_{0}^{y} \frac{\partial g}{\partial x}(x, \eta) d \eta \quad \text { for all } x \text { and } y
$$

and, for $0<\left|z^{\prime}\right|<|z|$,

$$
-\{f(x, y, z)-f(x, y, 0)\} y z \leqslant-y z^{2} \frac{\partial f}{\partial z}\left(x, y, z^{\prime}\right),
$$

by the Mean-value Theorem, we see readily that

$$
\begin{aligned}
\dot{U} \leqslant & -\delta_{2} x h(x)-\left[\left(y g(x, y)-b y^{2}\right)+\delta_{2} x(g(x, y)-b y)\right]- \\
& -\left(\delta_{1} f(x, y, z)-1\right) z^{2}-U_{1}-U_{2}-U_{3}-B z \chi(x)+ \\
& +\mid\left\{\delta_{2} x+(a+1) y+\left(1+\delta_{1} z\right\} p(t, x, y, z) \mid\right.
\end{aligned}
$$

where

$$
\left\{\begin{array}{l}
U_{1}=\operatorname{ayg}(x, y)-y^{2} h^{\prime}(x)-a \delta_{2} y^{2}+\frac{8 C}{\pi} y^{2} \chi^{\prime}(x) \\
U_{2}=\left\{b+\frac{8 C}{\pi \sqrt{2}} \delta_{1} \chi^{\prime}(x)-\delta_{1} h^{\prime}(x)\right\} y^{2} \\
U_{3}=(f(x, y, z)-a)\left(z^{2}+\delta_{2} x z\right)
\end{array}\right.
$$

The verification of (4.6) will be in two stages. First we show that there is a constant $D_{13}>\max \left(x_{0}, y_{0}\right)$ such that

$$
\dot{U} \leqslant-1 \text { provided } x^{2}+y^{2} \geqslant 2 D_{13}^{2} \text {. }
$$

Assume then, for a start, that $|y| \geqslant y_{0},|x| \geqslant x_{0}$; the other cases: $|y| \geqslant y_{0}$ and $|x| \leqslant x_{0}$, 
$|y| \leqslant y_{0}$ and $|x| \geqslant x_{0}$ will be treated separately. By hypothesis (i) of Theorem 2

$$
U_{3}=(f(x, y, z)-a)\left[\left(z^{2}+\frac{1}{2} \delta_{2} x\right)^{2}-\frac{1}{4} \delta_{2}^{2} x^{2}\right] \geqslant-\left(a^{\prime}-a\right) \frac{\delta_{2}^{2}}{4} x^{2} .
$$

Since (4.3) implies

$$
\chi^{\prime}(x) \geqslant 0 \text { for all } x \quad \text { and, } \quad \chi^{\prime}(x) \geqslant \frac{\pi \sqrt{2}}{8} \text { if }|x| \leqslant x_{0},
$$

we have, by (2.5) and (4.2), that

$$
U_{2} \geqslant\left(b-\delta_{1} c\right) y^{2} \quad \text { if }|x| \geqslant x_{0} \text { and, } U_{2} \geqslant b y^{2} \text { if }|x| \leqslant x_{0}:
$$

Therefore

$$
U_{2} \geqslant\left(b-\delta_{1} c\right) y^{2} \quad \text { for all } x \text { and } y \text {. }
$$

Similarly

$$
U_{1} \geqslant\left(a b-c-a \delta_{2}\right) y^{2} \quad \text { if }|x| \geqslant x_{0} \text { and }|y| \geqslant y_{0},
$$

by (1.5). Also, by (1.5), the term

$\left.\left[\left(y g(x, y)-b y^{2}\right)+\delta_{2} x g(x, y)-b y\right)\right]=(g(x, y) / y-b)\left\{\left(y+\frac{1}{2} \delta_{2} x\right)^{2}-\frac{1}{4} \delta_{2}^{2} x^{2}\right\} \geqslant$

$$
\geq-\frac{1}{4}\left(b^{\prime}-b\right) \delta_{2}^{2} x^{2}
$$

if $|y| \geqslant y_{0}$. Since $|\chi(x)| \leqslant 1, h(x) \mid x \geqslant \delta$ and $f(x, y, z) \geqslant a$, we have, on combining the above estimates with $(4.8)$, that

$$
\begin{aligned}
\dot{U} \leqslant-\left[\delta \delta_{2}-\frac{1}{4}\left(b^{\prime}-b\right) \delta_{2}^{2}-\frac{1}{4}\left(a^{\prime}-a\right) \delta_{2}^{2}\right] x^{2}- & {\left[\left(b-\delta_{1} c\right)+\left(a b-c-a \delta_{2}\right)\right] y^{2}-} \\
& -\left(a \delta_{1}-1\right) z^{2}+D_{14}\left(x^{2}+y^{2}+z^{2}\right)^{\frac{1}{3}}|p(t, x, y, z)|,
\end{aligned}
$$

the coefficients

$$
\left(a \delta_{1}-1\right),\left(b-\delta_{1} c\right),\left(a b-c-a \delta_{2}\right), \delta_{2}\left\{\delta-\frac{1}{4}\left(b^{\prime}-b\right) \delta_{2}-\frac{1}{4}\left(a^{\prime}-a\right) \delta_{2}\right\}
$$

being positive by (2.5). Thus by (1.7),

$$
\begin{aligned}
\dot{U} & \leqslant-D_{15}\left(x^{2}+y^{2}+z^{2}\right)+D_{14}\left(x^{2}+y^{2}+z^{2}\right)^{\frac{1}{2}}|p(t, x, y, z)| \\
& \leqslant-\left(D_{15}-D_{14} \Delta_{1}\right)\left(x^{2}+y^{2}+z^{2}\right)+\Delta_{0} D_{14}(|x|+|y|+|z|) \\
& \leqslant-\frac{1}{2} D_{15}\left(x^{2}+y^{2}+z^{2}\right)+\Delta_{0} D_{14}(|x|+|y|+|z|)
\end{aligned}
$$

provided that

$$
\Delta_{1} \leqslant \varepsilon_{1}, \varepsilon_{1}=\frac{1}{2} D_{15} D_{14}^{-1}
$$


Since $-\frac{1}{2} D_{15} z^{2}+\Delta_{0} D_{14}|z| \leqslant D$ for all $z$, we have that

$$
\dot{U} \leqslant-\frac{1}{2} D_{1 \bar{a}}\left(x^{2}+y^{2}\right)+A_{0} D_{14}(|x|+|y|)+D,
$$

from which it follows that there is a constant $D_{16}>\max \left(x_{0}, y_{0}\right)$ such that

$$
\dot{U} \leqslant-1 \text { provided } x^{2}+y^{2} \geqslant D_{16}^{2}
$$

In the case $|y| \geqslant y_{0},|x| \leqslant x_{0}$, the estimates (4.11) and (4.13) still hold for $U_{3}$ and $U_{2}$, but $U_{1}$ satisfies

$$
U_{1} \geqslant\left\{a b-a \delta_{2}+(\sqrt{2}-1) c\right\} y^{2}
$$

as can be verified from (4.9), (4.12) and (4.2). Thus, in this case, (4.8) yields

$$
\dot{U} \leqslant-\left(D_{17}-\Delta_{1} D_{14}\right)\left(y^{2}+z^{2}\right)+\Delta_{0} D_{14}(|y|+|z|)+D
$$

since $|h(x)|$ is bounded, where $D_{17}=\min \left[\left(a \delta_{1}-1\right),\left\{\left(b-\delta_{1} e\right)+a b-a \delta_{2}+(\sqrt{2}-1) C\right\}\right]$. The last inequality clearly implies

$$
\dot{U} \leqslant-\frac{1}{2} D_{17} y^{2}+D_{14} \Delta_{0}|y|+D
$$

provided $A_{1} \leqslant \varepsilon_{2}$, where

$$
\varepsilon_{2}=\frac{1}{2} D_{17} D_{14}^{-1}
$$

This shows that $\dot{U} \leqslant-1$ provided $|y|>y_{0}$ is large enough; that is, there exists $D_{18}>y_{0}$ such that

$$
\dot{U} \leqslant-1 \text { provided }|y| \geqslant D_{18} .
$$

It remains to consider the case $|y| \leqslant y_{0}$ and $|x| \geqslant x_{0}$. Here by (4.3) and (1.6), $-U_{1},-U_{2}$ are bounded, and since $\max |g(x, y)| \leqslant A$ it follows from (4.8) and (4.11) that

$$
\dot{U} \leqslant-\left(D_{19}-\Delta_{1} D_{14}\right)\left(x^{2}+z^{2}\right)+\Delta_{0} D_{14}|z|+D(|x|+1)
$$

where $D_{18}=\min \left\{\left(a \delta_{1}-1\right), \delta \delta_{2}-\left(a^{\prime}-a\right) \delta_{2}^{2} / 4\right\}$. Now let

$$
\varepsilon_{3}=\frac{1}{2} D_{19} D_{14}^{-1}
$$

then provided $A_{1} \leqslant \varepsilon_{3}$, it follows as in the preceding ease that there is a constant $D_{20}>x_{0}$ such that

$$
\dot{U} \leqslant-1 \text { provided }|x| \geqslant D_{20}
$$


Now set

$$
\varepsilon_{4}=\min \left(\varepsilon_{1}, \varepsilon_{2}, \varepsilon_{3}\right)
$$

where $\varepsilon_{1}, \varepsilon_{2}, \varepsilon_{3}$ are given by (4.14), (4.16) and (4.18) respectively. Then if $\Delta_{1} \leqslant \varepsilon_{4}$,

$$
\ddot{U} \leqslant-1 \text { provided } x^{2}+y^{2} \geqslant D_{16}^{2}+D_{18}^{2}+D_{20}^{2}
$$

and this verifies $(4.10)$ with $2 D_{13}^{2}=D_{16}^{2}+D_{18}^{2}+D_{20}^{2}$ :

To conclude the proof of $(4.6)$ we shall now show that there is a constant $D_{21}$ such that

$$
\dot{U} \leqslant-1 \text { when }|x| \leqslant x_{0} \text { and }|y| \leqslant y_{0} \text { provided that }|z| \geqslant D_{21} \text {. }
$$

Indeed let $|x| \leqslant x_{0}$ and $|y| \leqslant y_{0}$. Then since $-U_{1},-U_{2}$ are bounded and $U_{3}$ sa. tisfies (4.11), it will be clear from (4.8) and (1.7) that $\dot{U}$ satisfies

$$
\dot{U} \leqslant-\left(D_{22}-\Delta_{1} D_{14}\right) z^{2}+D(|z|+1)
$$

where $D_{22}=a \delta_{1}-1$. Thus, if

$$
\begin{gathered}
\Delta_{1} \leqslant \varepsilon_{5}, \varepsilon_{5} \equiv \frac{1}{2} D_{22} D_{14}^{-1}, \\
\dot{U} \leqslant-\frac{1}{2} D_{22} z^{2}+D(|z|+1) \\
\leqslant-1
\end{gathered}
$$

provided $|z|$ is sufficiently large, say $|z| \geqslant D_{23}$. This verifies (4.21).

The two results (4.10) and (4.21) show clearly that if $\Delta_{1} \leqslant \varepsilon, \varepsilon=\min \left(\varepsilon_{4}, \varepsilon_{5}\right)$ then

$$
\dot{U} \leqslant-1 \text { provided that } x^{2}+y^{2}+z^{2}>2 D_{13}^{2}+D_{21}^{2} \text {, }
$$

and this verifies (4.6) with $D_{11}=2 D_{13}^{2}+D_{21}^{2}$.

Theorem 2 now follows as was pointed out with $\varepsilon=\min \left(\varepsilon_{4}, \varepsilon_{5}\right)$.

\section{REFERENCES}

[1] J. O. C. Ezerco, Ann. Mat. Pura Appl, (IV), 66 (1964), pp. 233-250.

[2] J. O. C. EzexLo, J. London Math. Soc., 38 (1963), pp. 11-16.

[3] M. Harrow, J. London. Math. Soc., 43 (1968), pp. 587-592.

[4] R. Reissig, Math. Nachr., 32 (1966), pp. 83-88.

[5] H. O. Tejomola, Ann. Mat. Pura Appl., (IV), 83 (1969), pp. 195-212. 